\title{
Theory of emittance exchange through coupling resonance crossing
}

\author{
Masamitsu Aiba $\odot$ and Jonas Kallestrup $\odot$ \\ Paul Scherrer Institut, 5232, Villigen, Switzerland
}

(Received 11 December 2019; accepted 23 April 2020; published 30 April 2020)

\begin{abstract}
In circular accelerators, transverse beam emittances can be exchanged by means of a coupling resonance crossing. It was first demonstrated experimentally at CERN accelerators, before a theoretical study revealed its mechanism. The existing theory of emittance exchange seems, however, to be deficient. In this study, we establish a theory of emittance exchange using the matrix formalism. Furthermore, we have found that the mechanism of emittance exchange is analogous to the transition dynamics of two-state quantum systems, thereby allowing the Landau-Zener formula to be applied for the prediction of transverse emittance values after nonadiabatic crossing.
\end{abstract}

DOI: 10.1103/PhysRevAccelBeams.23.044003

\section{INTRODUCTION}

Emittance exchange is one of a number of beam manipulation techniques in accelerators. Two particularly useful applications in lepton machines are known: at free electron laser linear accelerators, where the exchange of longitudinal and transverse emittances suppresses beam deterioration due to longitudinal beam instabilities [1,2], and at the booster synchrotron injector of light source facilities, to facilitate beam injection into the storage ring $[3,4]$. The latter application is becoming increasingly relevant owing to several planned next generation synchrotron light sources, where beam injection is of critical importance [5]. In hadron machines, the technique is applied to beam emittance measurements [6]. More studies related to the emittance exchange (or manipulation) are found elsewhere [7-9]. The beam dynamics of emittance exchange in circular accelerators is the subject of the present work.

The transverse beam particle motion is characterized by the so-called betatron tunes, which are the number of oscillations per revolution in the horizontal and vertical planes, $Q_{x}$ and $Q_{y}$, respectively. Linear coupling resonances, $Q_{x} \pm Q_{y}=l$, where $l$ is integer, may significantly affect the behavior of beam particles in circular accelerators. Even when the resonance sources, i.e., the skew quadrupole and solenoid field components, are weak, their effect on the beam is greatly enhanced when the working point $\left(Q_{x}, Q_{y}\right)$ is close to the resonance. This was closely studied by, among others, Guignard [10] for the case of a

Published by the American Physical Society under the terms of the Creative Commons Attribution 4.0 International license. Further distribution of this work must maintain attribution to the author(s) and the published article's title, journal citation, and DOI. stationary working point, i.e., where the betatron tunes are constant over time. With regard to the difference coupling resonance, $Q_{x}-Q_{y}=l$, the equations of motion, including coupling terms, were analyzed, to conclude that the horizontal and vertical emittance values oscillate with opposite phase [11]. The fraction of the energy of the betatron oscillation interchanged between the transverse planes, $F$, was found to be

$$
F=\frac{|C|^{2}}{\Delta^{2}+|C|^{2}}
$$

where $C$ is the coupling coefficient, and $\Delta$ is the detuning of the working point with respect to the resonance condition $\left(Q_{x}-Q_{y}+\Delta=l\right)$. When $\Delta=0$, for instance on resonance, the horizontal and vertical emittances are fully exchanged at corresponding phases periodically.

Knowing the emittance oscillation, we can exchange the transverse beam emittances, for example, by injecting a beam into a ring where the resonance condition is exactly met, and subsequently extracting the beam when the emittances are exchanged. Another approach would be to use a pulsed skew quadrupole to excite the resonance for a short time until the exchange is complete [3]. On the other hand, it was found experimentally at CERN accelerators (Intersecting Storage Ring, Antiproton Accumulator, Proton Synchrotron and Proton Synchrotron Booster) that an emittance exchange is also realized when the working point is moved across a resonance [6]. This approach would be far more practical than those previously mentioned. A theoretical study followed to reveal the mechanism of exchange [12].

In [12] at first a simple model is introduced: when the working point crosses a resonance adiabatically, the beam emittances vary during the crossing as 


$$
\begin{aligned}
& \epsilon_{x}=\epsilon_{x 0} \cos ^{2} \theta+\epsilon_{y 0} \sin ^{2} \theta, \\
& \epsilon_{y}=\epsilon_{x 0} \sin ^{2} \theta+\epsilon_{y 0} \cos ^{2} \theta,
\end{aligned}
$$

where $\epsilon_{x 0, y 0}$ are the initial horizontal and vertical emittances, respectively. $\theta$ is the angle between the laboratory frame axis and the normal mode axis, where the motion is decoupled [13]. We interpret these equations that, when $\theta$ changes from zero to $\frac{\pi}{2}$, the emittance is fully exchanged.

However, the range of $\theta$ is not clearly defined. In the course of the derivation in [12], the parameters $|C|$ and $\Delta$ are related to $\theta$ as

$$
\cos 2 \theta=\cos \left(\arctan \frac{|C|}{\Delta}\right)=\frac{1}{\sqrt{1+|C|^{2} / \Delta^{2}}} .
$$

This clearly leads to an inequality of the form $-\frac{\pi}{4} \leq \theta \leq \frac{\pi}{4}$, i.e., the emittance can be equalized but not exchanged whereas the final result [Eq. (34) in [12]] implicitly assumes a range of $0 \leq \theta \leq \frac{\pi}{2}$ or $-\frac{\pi}{2} \leq \theta \leq 0$.

Then the emittance exchange is explained using the resonance driving term formalism, which describes the accelerator in a more comprehensive manner. In this refined model, the emittance exchange originates in an invariant exchange of a single particle that is induced by a resonance crossing.

We, however, have a different picture based on our new theory of emittance exchange, which is described in Sec. II. Moreover, these theories are restricted to adiabatic crossings. We establish, in Sec. III, an analytical equation that quantifies the emittance values after crossing, including crossing speed as a parameter. Finally we draw our conclusions in Sec. IV.

\section{THEORY BASED ON MATRIX FORMALISM (ADIABATIC CROSSING)}

We find that the emittance exchange can be well described by using the matrix formalism defined in [14]. We import here some relevant equations and statements from [14].

The linear transverse motion is described by the socalled one-turn map, a $4 \times 4$ matrix,

$$
\mathbf{T}=\left(\begin{array}{ll}
\mathbf{M} & \mathbf{m} \\
\mathbf{n} & \mathbf{N}
\end{array}\right)
$$

The off-diagonal $2 \times 2$ block matrices are (non)zero with (non)zero coupling source. The one-turn map can be block diagonalized through a similarity transformation [15],

$$
\mathbf{T}=\mathbf{V U V}^{-1}
$$

We get

$$
\mathbf{U}=\left(\begin{array}{ll}
\mathbf{A} & \mathbf{0} \\
\mathbf{0} & \mathbf{B}
\end{array}\right)
$$

The submatrices $\mathbf{A}$ and $\mathbf{B}$ represent two eigenmodes (or normal modes). The matrix $\mathbf{V}$ is expressed as

$$
\mathbf{V}=\left(\begin{array}{cc}
\gamma \mathbf{I} & \mathbf{C} \\
-\mathbf{C}^{+} & \gamma \mathbf{I}
\end{array}\right)
$$

where the superscript " + " indicates symplectic conjugate [14]. The solution for $\gamma$ and $\mathbf{C}$ is

$$
\gamma=\sqrt{\frac{1}{2}+\frac{1}{2} \sqrt{\frac{(\operatorname{Tr}[\mathbf{M}-\mathbf{N}])^{2}}{(\operatorname{Tr}[\mathbf{M}-\mathbf{N}])^{2}+4 \operatorname{det} \mathbf{H}}}}
$$

with $\mathbf{H}=\mathbf{m}+\mathbf{n}^{+}$, and

$$
\mathbf{C}=\frac{-\mathbf{H} \operatorname{sgn}(\operatorname{Tr}[\mathbf{M}-\mathbf{N}])}{\gamma \sqrt{(\operatorname{Tr}[\mathbf{M}-\mathbf{N}])^{2}+4 \operatorname{det} \mathbf{H}}}
$$

The coordinates (particle position and angle) of the laboratory frame and the normal mode are then related as follows:

$$
\left(a, a^{\prime}, b, b^{\prime}\right)^{T}=\mathbf{V}^{-1}\left(x, x^{\prime}, y, y^{\prime}\right)^{T} .
$$

In the matrix formalism, the normal mode tunes, $Q_{A}$ and $Q_{B}$, are found from $\mathbf{A}$ and $\mathbf{B}$ matrices. $|C|$ of Eq. (1) is then found from these values computed on the coupling resonace as $\left|Q_{A}-Q_{B}\right|$.

For $\operatorname{det} \mathbf{H}>0$, a second solution exists,

$$
\gamma=\sqrt{\frac{1}{2}-\frac{1}{2} \sqrt{\frac{(\operatorname{Tr}[\mathbf{M}-\mathbf{N}])^{2}}{(\operatorname{Tr}[\mathbf{M}-\mathbf{N}])^{2}+4 \operatorname{det} \mathbf{H}}}}
$$

with $\mathbf{H}=\mathbf{m}+\mathbf{n}^{+}$, and

$$
\mathbf{C}=\frac{\mathbf{H} \operatorname{sgn}(\operatorname{Tr}[\mathbf{M}-\mathbf{N}])}{\gamma \sqrt{(\operatorname{Tr}[\mathbf{M}-\mathbf{N}])^{2}+4 \operatorname{det} \mathbf{H}}}
$$

Use of the first or second solution is arbitrary when $\operatorname{det} \mathbf{H}>0$. (They are referred to as solution I and solution II hereafter.) When the coupling is weak, $\mathbf{V}^{-1}$ in Eq. (11) is approximately a unit matrix for solution I and an off blockdiagonal matrix for solution II. It is therefore natural to take solution I such that the horizontal and vertical particle motions are associated to $\mathbf{A}$ and $\mathbf{B}$, or simply $\mathrm{A}$ and $\mathrm{B}$ modes, respectively. A switch in solutions, however, may be necessitated particularly when det $\mathbf{H}$ changes sign along the ring. The associations of the normal mode coordinates to the laboratory frame coordinates are interchanged 
between the two solutions, leading to the term "mode flipping."

We now imagine a virtual ring that consists of many original rings connected to one another (Fig. 1). This virtual ring is henceforth referred to as the "large ring," while the ring under investigation is referred to as the "original ring." The original ring includes some coupling sources, and the coupling resonance close to the working point is excited only modestly, such that the mode flipping does not appear within the original ring. The dynamic feature of the resonance crossing is introduced as follows. Each segment of the large ring, which corresponds to one revolution in the original ring, has different betatron phase advances such that the working point of the original ring crosses a coupling resonance. Another condition is introduced as the working point of the original ring returns to the initial point along the large ring. This means that the working point of the original ring crosses the resonance twice along the large ring. Just to avoid potential confusion, we add a superscript " $L$ " to the symbols of the large ring in the following.

When the variation of the phase advance from one segment to the next is infinitesimally small, all the elements of the original-ring one-turn maps are smoothly connected. At the same time, the elements of matrices $\mathbf{A}$ and $\mathbf{B}$ of those one-turn maps have to be smoothly connected to form a periodic condition in the large ring. In other words, such a periodic condition is the solution that the beam follows. Owing to the fact that $\operatorname{Tr}[\mathbf{M}-\mathbf{N}]$ changes sign when the working point of the original ring crosses the resonance, we have to switch from one solution to the other, otherwise the elements of matrix $\mathbf{C}$ are discontinuous [Eqs. (10) and (13)]. This ensures the smooth connections of $\mathbf{A}$ and $\mathbf{B}$ matrix elements as well, since these matrices are functions of $\gamma, \mathbf{M}, \mathbf{N}, \mathbf{m}, \mathbf{n}$ and $\mathbf{C}$. We have $\gamma=1 / \sqrt{2}$ for both solution I and II when $\operatorname{Tr}[\mathbf{M}-\mathbf{N}]=0$, and thus it is also continuous over the switching. The necessity of the switching indicates that the large ring includes mode flippings at

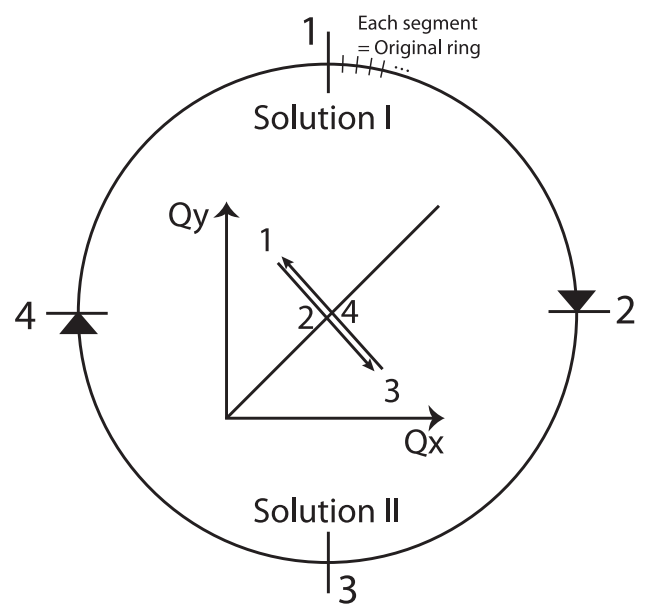

FIG. 1. "Large ring" that consists of many original rings. The working point of the original ring is also shown. the locations where the working point of the original ring crosses the resonance, namely locations 2 and 4 in Fig. 1.

We simulate a simple large ring where the original ring (or each segment) consists of a zero-coupling one-turn map multiplied by a thin skew quadrupole matrix. The working point of the original ring is controlled by changing the tunes of the zero-coupling one-turn map. It is then straightforward to numerically generate a one-turn map of the large ring. In [14], it is mentioned that a mode flipping can be detected by checking the trace of $\mathbf{A}$ and $\mathbf{B}$ along the ring. We thus observe the traces of $\mathbf{A}^{L}$ and $\mathbf{B}^{L}$ along the large ring. Figure 2 shows the traces and the parameter $\gamma^{L}$ along the large ring.
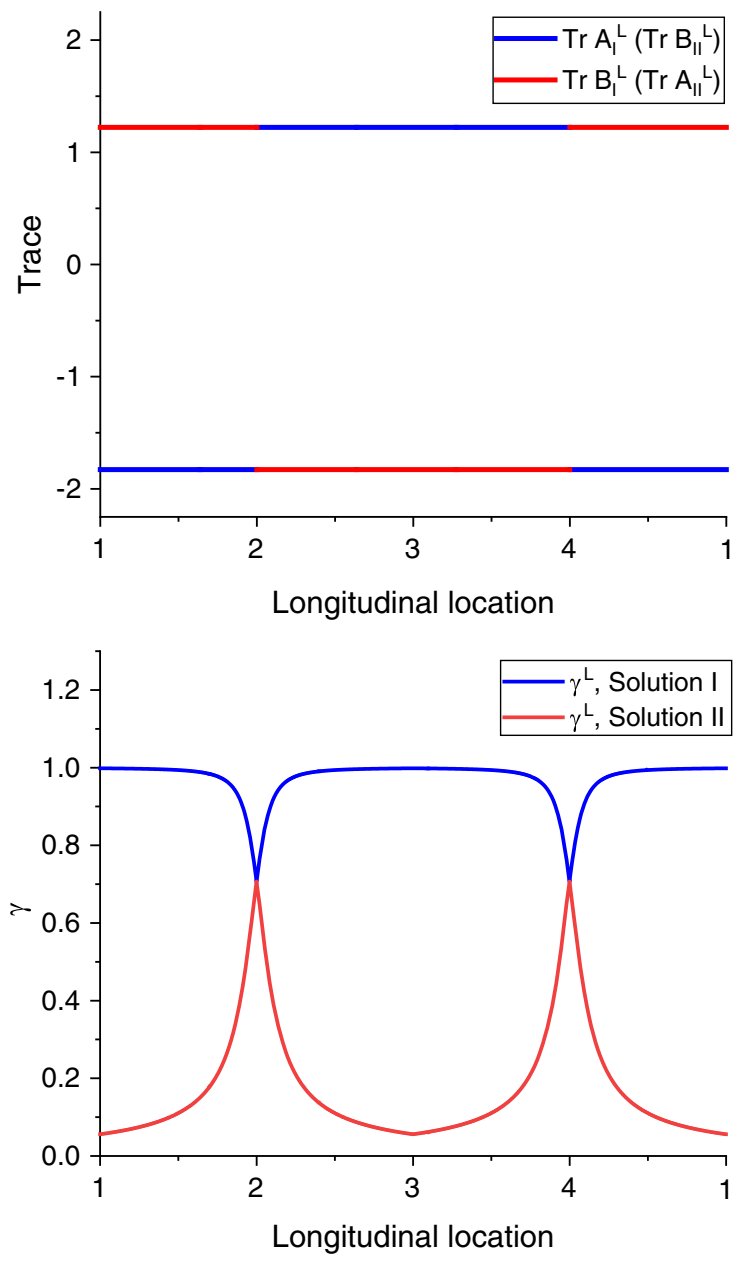

FIG. 2. Traces of $\mathbf{A}^{L}$ and $\mathbf{B}^{L}$ matrices (top) and the parameter $\gamma^{L}$ (bottom) of the large ring one-turn map. It is noted that $\operatorname{Tr} \mathbf{A}$ of solution I(II) is equal to $\operatorname{Tr} \mathbf{B}$ of solution II(I). The betatron tunes of the original ring are varied in a range of $Q_{x}=0.3 \mp 0.005$ and $Q_{y}=0.3 \pm 0.005$. The resonance condition, $Q_{x}=Q_{y}$, is fulfilled at locations 2 and 4 . The beta functions of the original rings are set to arbitrary values $\left(\beta_{x}=10 \mathrm{~m}, \beta_{y}=5 \mathrm{~m}\right)$ and constant. The integrated, normalized skew quadrupole strength is set to $0.001 \mathrm{~m}^{-1}$, corresponding to $|C| \sim 0.001$. The large ring consists of $400000+1$ original rings, ensuring smooth connections of one-turn maps and avoiding an integer resonance. 
In Fig. 2, as we expect, the traces of the $\mathbf{A}^{L}$ and $\mathbf{B}^{L}$ matrices are interchanged at the longitudinal locations 2 and 4 , where the working point of the original ring crosses the resonance correspondingly. Furthermore, this is not observed when the skew quadrupole is turned off or when the working point is moved but does not cross the resonance. From this investigation, we understand how the emittance exchange occurs: during the crossing, the mode flipping occurs at the resonance, and thus the particle motion associated to the horizontal plane before the crossing is associated to the vertical plane after the crossing, and vice versa. If we notice that the parameter $\gamma$ is related to the angle between the laboratory frame axis and the normal mode axis as $\theta=\arccos \gamma$, we get a further simple picture, i.e., the normal mode axis is rotating from $\theta=0$ to $\frac{\pi}{2}$ during the resonance crossing (or $-\frac{\pi}{2}$ depending on the direction of crossing). When the rotation is slow enough, i.e., adiabatic, all beam particles keep their oscillation amplitudes on the normal mode axes. The mode flipping is a consequence of the fact that the angle $\theta$ crosses $\frac{\pi}{4}$ (or $-\frac{\pi}{4}$ ).

Our model thus supports the simple model in [12] but the range of $\theta$ and how it varies during the crossing are now clearly defined. On the other hand, the mode flipping is simply a matter of the coordinate system. We think that the invariant exchange described in [12] is equivalent to the mode flipping and thus not a physical phenomenon.

\section{NONADIABATIC CROSSING}

A similar exercise to that of Fig. 2 is performed for the original-ring one-turn map. The traces of $\mathbf{A}$ and $\mathbf{B}$ are computed for various working points. In Fig. 3, the normal mode tunes are plotted instead of trace values. It is seen that the tunes are smoothly connected at the resonance when we switch from one solution to the other. Figure 3 together with Fig. 2 also gives us an insight into the underlying physics: the normal mode tunes of the coupled oscillation vary smoothly and follow the curves of Fig. 3 as long as the crossing is adiabatically slow.

Note that in Fig. 3, solutions I and II are explicitly labeled in addition to modes A and B. Unfortunately, the labeling in the existing literature is not always done in a consistent manner. Some authors place $Q_{A}$ at the bottomleft and top-right of the diagram, and $Q_{B}$ elsewhere, while others place only one of $Q_{A}$ and $Q_{B}$ for a given curve. All permutations remain "correct" owing to the fact that solutions I and II may be chosen arbitrarily. When discussing a crossing where the parameter $\Delta$ is varying with time, however, it is essential to distinguish not only between modes A and B, but also solutions I and II.

The question naturally arises as to how slow a crossing should be in order to be adiabatic. To answer this question, we turn to the Landau-Zener formula (LZF) [16,17], which was originally developed to study the transition of molecule states between two energy levels [17]. It has since been applied to various kinds of problems in physical chemistry,

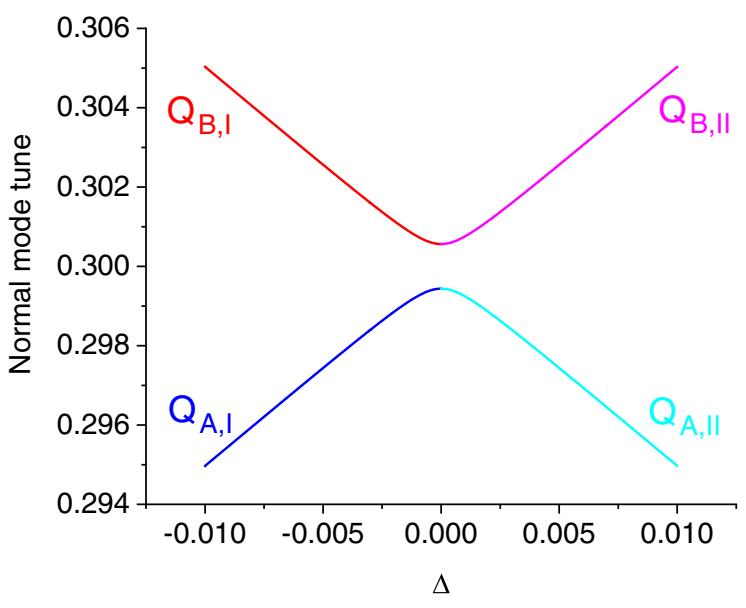

FIG. 3. Normal mode tunes of the original ring one-turn map around the resonance. The parameters are the same as those in Fig. 2. In this plot, solution I is used for $\Delta<0$ and solution II for $\Delta>0$.

particle physics, spin dynamics, photonics and elsewhere [18-23].

The diagram of Fig. 3 is generically referred to as "avoided crossing." By analogy, the minimum energy gap of the avoided crossing, equal to $|C|$, is referred to as "closest tune" in the accelerator field. The two energy levels, $E_{1}$ and $E_{2}$, are equivalent to the normal mode tunes, $Q_{A}$ and $Q_{B}$, since energy is related to frequency through the Planck constant.

The LZF is an analytical solution of the transition probability for a nonadiabatic crossing, $P_{n a}$, expressed as

$$
P_{n a}=\exp \left(-2 \pi \frac{\left|H_{12}\right|^{2}}{\hbar|\alpha|}\right)
$$

where $\hbar$ is the reduced Planck constant, $\left|H_{12}\right|$ is half the minimum energy gap, and $\alpha$ is a constant representing the crossing speed. Here we follow the notation used in [24]. $P_{n a}$ asymptotically approaches zero (no transition) as $|\alpha|$ decreases.

It was derived by solving the time-dependent Schrödinger equation for a wave function, linearly combining two states, $\phi_{1}$ and $\phi_{2}$, associated to $E_{1}$ and $E_{2}$,

$\psi=A \phi_{1} \exp \left(-i \int{ }^{t} E_{1} d t\right)+B \phi_{2} \exp \left(-i \int{ }^{t} E_{2} d t\right)$.

This yields a set of second-order differential equations,

$$
\begin{aligned}
& \ddot{A}-i E_{12} \dot{A}+\left|H_{12}\right|^{2} A=0, \\
& \ddot{B}+i E_{12} \dot{B}+\left|H_{12}\right|^{2} B=0,
\end{aligned}
$$

where $E_{12}=E_{2}-E_{1}$, and $\dot{A}=d A / d t$, etc. Replacing $E_{12}$ by $\alpha t$ and solving Eq. (17) leads to Eq. (14). While for a 


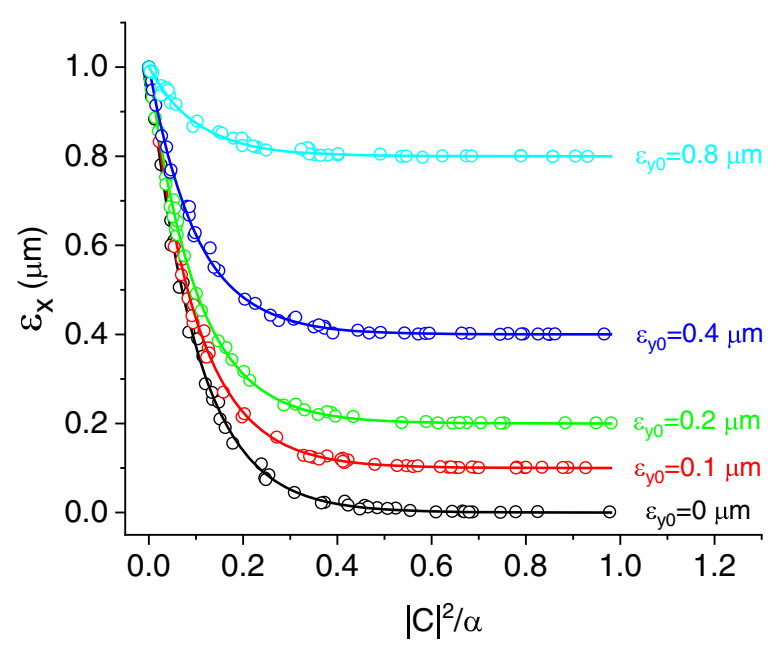

FIG. 4. Horizontal emittance after resonance crossing. Open circles are from tracking simulations, lines are from Eq. (18). The initial horizontal emittance is always $1 \mu \mathrm{m}$ while the initial vertical emittances are 0/0.1/0.2/0.4/0.8 $\mu \mathrm{m}$ (black/red/lightgreen/blue/cyan).

wave function like Eq. (15) the physical meaning lies in $E_{12}$, by analogy, for beam particles close to a coupling resonance it is the difference $Q_{A}-Q_{B}$ that matters rather than the absolute values of $Q_{A}$ and $Q_{B}$. Moreover, Eq. (17) is identical to Eq. (1.5.15) in [10] including the meaning of coefficients, where the particle motion is analyzed through the equations of motion of a charged particle in a magnetic field. The problem tackled by the LZF is equivalent to our emittance exchange problem.

$P_{n a}$ corresponds, in our case, to a fraction of the initial betatron action of each particle that remains after crossing, and the rest goes to the other plane. For an infinitely slow crossing, $P_{n a}$ is zero, and thus the initial horizontal (vertical) action is fully transferred to the final vertical (horizontal) action. $P_{n a}$ here no longer means probability that describes the quantum phenomena but it determines the final action of the betatron motion, which is a continuous variable. Finally, for a beam that is an ensemble of many particles, the beam emittances after crossing are obtained as

$$
\begin{aligned}
& \epsilon_{x}=P_{n a} \epsilon_{x 0}+\left(1-P_{n a}\right) \epsilon_{y 0}, \\
& \epsilon_{y}=\left(1-P_{n a}\right) \epsilon_{x 0}+P_{n a} \epsilon_{y 0},
\end{aligned}
$$

with

$$
P_{n a}=\exp \left(-\pi^{2} \frac{|C|^{2}}{|\dot{\Delta}|}\right)
$$

where $\dot{\Delta}$ is the change of $\Delta$ per revolution in the original ring [replacing $\alpha$ of Eq. (14)]. The Planck constant, $h=2 \pi \hbar$, is set to one given that we take $Q_{A}$ and $Q_{B}$ to be equivalent to $E_{1}$ and $E_{2}$. Equation (18) is confirmed through a particle tracking simulation based on the aforementioned one-turn maps (Fig. 4).

In our previous work [4], it was shown that $\mathcal{S}=\frac{\dot{\Delta}}{|C|^{2}}$ was a scaling factor to describe the quality of emittance exchange. From numerical tracking, it was found that the emittances were almost fully exchanged, i.e., adiabatic, when $\mathcal{S}<3$. In the present work, we understand that this condition corresponds to $P_{n a}$ close to zero $\left[P_{n a}<\exp \left(-\pi^{2} / 3\right)=0.037\right]$.

\section{CONCLUSION}

Emittance exchange through linear coupling resonance crossing is studied. We established a theory of emittance exchange using the matrix formalism for adiabatic crossings. It was shown in our theory that the emittance exchange is a consequence of the rotation of the normal mode axis by approximately $\frac{\pi}{2}$ radian. Furthermore, we have found that the mechanism of emittance exchange is analogous to the transition dynamics of two-state quantum systems. Therefore, we can predict transverse emittance values after nonadiabatic crossing by applying the LandauZener formula with the relevant accelerator parameters. The theoretical prediction is confirmed by numerical tracking.

\section{ACKNOWLEDGMENTS}

The authors are grateful to Michael Böge and Bernard Riemann for valuable discussions and to Jan Chrin and Thomas Schietinger for proofreading the manuscript.

[1] P. Emma, Z. Huang, K.-J. Kim, and P. Piot, Transverse-tolongitudinal emittance exchange to improve performance of high-gain free-electron lasers, Phys. Rev. ST Accel. Beams 9, 100702 (2006).

[2] G. Ha et al., Precision Control of the Electron Longitudinal Bunch Shape Using an Emittance-Exchange Beam Line, Phys. Rev. Lett. 118, 104801 (2017).

[3] P. Kuske and F. Kramer, Transverse emittance exchange for improved injection efficiency, in Proceedings of the 7th International Particle Accelerator Conference (JACoW Publishing, Geneva, 2016), pp. 2028-2031.

[4] J. Kallestrup and M. Aiba, Emittance exchange in electron booster synchrotron by coupling resonance crossing, Phys. Rev. Accel. Beams 23, 020701 (2020).

[5] R. Hettel, Challenges in the design of diffraction-limited storage rings, in Proceedings of the 5th International Particle Accelerator Conference (JACoW Publishing, Geneva, 2014), pp. 7-11.

[6] C. Carli and G. Cyvoct, Emittance exchange by crossing a coupling resonance: A method for precise measurements of the horizontal emittance applied to the PSB, CERN Report No. PS/AE/Note-2001-018, 2001.

[7] M. Hub, The exchange of the transverse phase planes in the injection beam transfer channel to the $300 \mathrm{GeV}$ main ring, CERN Report No. LABII-BT-72-2, 1972. 
[8] A. W. Chao and P. Raimondi, Emittance adapter for a diffraction limited synchrotron radiation source, SLAC Report No. 14808, 2012.

[9] G. Franchetti and I. Hofmann, Space charge effect on emittance exchange by skew quadrupoles, in Proceedings of the 18th Particle Accelerator Conference, New York, 1999 (IEEE, Piscataway, NJ, 1999), pp. 1782-1784.

[10] G. Guignard, The general theory of all sum and difference resonances in a three-dimensional magnetic field in a synchrotron, CERN Report No. CERN-76-06, 1976.

[11] G. Guignard, Beam blow-up and luminosity reduction due to linear coupling, CERN Report No. CERN-ISR-BOM/ 77-43, 1977.

[12] A. Franchi, E. Metral, and R. Tomás, Emittance sharing and exchange driven by linear betatron coupling in circular accelerator, Phys. Rev. ST Accel. Beams 10, 064003 (2007).

[13] In [12], the angle is denoted by $\alpha$. We replace it here by $\theta$ since we use $\alpha$ in Sec. III for a different parameter.

[14] D. Sagan and D. Rubin, Linear analysis of coupled lattices, Phys. Rev. ST Accel. Beams 2, 074001 (1999).

[15] D. A. Edwards and L. C. Teng, Parametrization of linear coupled motion in periodic systems, IEEE Trans. Nucl. Sci. 20, 885 (1973).

[16] L. Landau, A theory of energy transfer. II, Collected Papers of L.D. Landau (Pergamon, New York, 1965), pp. 52-59, https://doi.org/10.1016/B978-0-08-0105864.50014-6.

[17] C. Zener, Nonadiabatic crossing of energy levels, Proc. R. Soc. A 137, 696 (1932).

[18] E. E. Nikitin, Nonadiabatic transitions: What we learned from old masters and how much we owe them, Annu. Rev. Phys. Chem. 50, 1 (1999).

[19] H. Kubo, F. C. Jundt, and K. H. Purser, Application of Landau-Zener formula for the ratios of target to projectile K-shell vacancy production, J. Phys. Soc. Jpn. 37, 1612 (1974).

[20] C. W. Kim, W. K. Sze, and S. Nussinov, Neutrino oscillations and the Landau-Zener formula, Phys. Rev. D 35, 4014 (1987).

[21] M. Froissart and R. Stora, Depolarisation d'un faisceau de protons polarises dans un synchrotron, Nucl. Instrum. Methods 7, 297 (1960).

[22] J. R. Petta, H. Lu, and A. C. Gossard, A coherent beam splitter for electronic spin states, Science 327, 669 (2010).

[23] H. Suchowski, G. Porat, and A. Arie, Adiabatic processes in frequency conversion, Laser Photonics Rev. 8, 333 (2014).

[24] C. Wittig, The Landau-Zener formula, J. Phys. Chem. B 109, 8428 (2005). 